\title{
A Consecutive Approach to Alkenes that Combines Radical Addition of Phosphorus Hydrides with Horner-Wadsworth-Emmons-type Reactions
}

\author{
Mark P. Healy, Andrew F. Parsons and James G.T. Rawlinson
}

Representative example of radical addition of a phosphorus hydride to an alkene or diene: $O, O$-diethyl octylphosphonothioate (1)

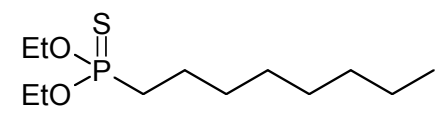

1-Octene (1.000 g, $8.18 \mathrm{mmol}, 1$ eq.), diethyl thiophosphite (3.793 g, $24.5 \mathrm{mmol}, 3$ eq.) and $\mathrm{Et}_{3} \mathrm{~B}(2.46 \mathrm{~mL}, 2.46 \mathrm{mmol}, 0.3$ eq.) were stirred in cyclohexane (40 mL) at r.t. under $\mathrm{N}_{2}$. Further portions of $\mathrm{Et}_{3} \mathrm{~B}(2.46 \mathrm{~mL}, 2.46 \mathrm{mmol}, 0.3$ eq.) were added at 1 $\mathrm{h}$ intervals until 1.5 eq. of $\mathrm{Et}_{3} \mathrm{~B}$ had been added in total (each addition of $\mathrm{Et}_{3} \mathrm{~B}$ was followed by addition of oxygen, this was done by opening the reaction vessel to air for 15 seconds before resealing). The solution was stirred overnight then concentrated in vacuo. A Kugelrohr distillation was carried out at $75^{\circ} \mathrm{C} / 3 \mathrm{mmHg}$ to remove excess diethyl thiophosphite as the distillate. Purification of the residue by column chromatography (silica, petrol/EtOAc, 19:1) afforded the title compound $\mathbf{1}$ (2.152 g, 99\%) as a colourless oil. $\mathrm{R}_{\mathrm{f}} 0.45$ (petrol/EtOAc, 9:1); $v_{\max }$ (neat) 2982 (s), 2954 (s), 2856 (s), 2364 (w), 1465 (m), 1388 (m), 1160 (m), 1097 (s), 1028 (P-OEt, s) cm ; $\delta_{\mathrm{H}}$ (400 MHz; $\left.\mathrm{CDCl}_{3}\right)$ 4.19-3.97 (4H, m, $\left.2 \times \mathrm{OCH}_{2} \mathrm{CH}_{3}\right), 1.92-1.84\left(2 \mathrm{H}, \mathrm{m}, \mathrm{PCH}_{2}\right), 1.63-$ $1.51\left(2 \mathrm{H}, \mathrm{m}, \mathrm{PCH}_{2} \mathrm{CH}_{2}\right), 1.42-1.14\left(10 \mathrm{H}, \mathrm{m}, 5 \times \mathrm{CH}_{2}\right), 1.30(6 \mathrm{H}, \mathrm{t}, J=7.0,2 \times$ $\left.\mathrm{OCH}_{2} \mathrm{CH}_{3}\right), 0.84\left(3 \mathrm{H}, \mathrm{t}, J=7.0, \mathrm{CH}_{2} \mathrm{CH}_{2} \mathrm{CH}_{3}\right) ; \delta_{\mathrm{C}}\left(100 \mathrm{MHz} ; \mathrm{CDCl}_{3}\right) 62.2(\mathrm{~d}, J=7.0$, $\left.2 \times \mathrm{OCH}_{2} \mathrm{CH}_{3}\right), 34.6\left(\mathrm{~d}, J=111.0, \mathrm{PCH}_{2}\right), 31.8\left(\mathrm{CH}_{2}\right), 30.3(\mathrm{~d}, J=18.5$, $\left.\mathrm{PCH}_{2} \mathrm{CH}_{2} \mathrm{CH}_{2}\right), 29.1\left(2 \times \mathrm{CH}_{2}\right), 22.7\left(\mathrm{~d}, J=6.0, \mathrm{PCH}_{2} \mathrm{CH}_{2}\right), 22.6\left(\mathrm{CH}_{2} \mathrm{CH}_{2} \mathrm{CH}_{3}\right), 16.2$ (d, $\left.J=7.0,2 \times \mathrm{OCH}_{2} \mathrm{CH}_{3}\right), 14.1\left(\mathrm{CH}_{2} \mathrm{CH}_{2} \mathrm{CH}_{3}\right) ; m / z\left(\mathrm{CI}, \mathrm{NH}_{3}\right) 267\left(\mathrm{M}+\mathrm{H}^{+}, 100 \%\right)$; (Found: $\mathrm{M}+\mathrm{H}^{+}, 267.1546 . \mathrm{C}_{12} \mathrm{H}_{27} \mathrm{O}_{2} \mathrm{PS}$ requires: $\mathrm{M}+\mathrm{H}^{+}, 267.1548$ ).

Representative example of a HWE reaction of a phosphonothioate: 9-methyl-8icosene (2b) 


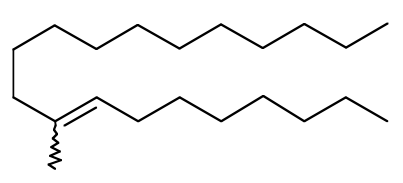

To a solution of $O, O$-diethyl octylphosphonothioate 1 ( $0.250 \mathrm{~g}, 0.94 \mathrm{mmol}, 1$ eq. $)$ in dry THF $(20 \mathrm{~mL})$ at $-78{ }^{\circ} \mathrm{C}$, under $\mathrm{N}_{2}$, was added ${ }^{\mathrm{s}} \mathrm{BuLi}(1.49 \mathrm{~mL}, 1.26 \mathrm{M}$ in cyclohexane, $1.88 \mathrm{mmol}, 2$ eq.). The solution was allowed to warm to $0{ }^{\circ} \mathrm{C}$ then cooled to $-78{ }^{\circ} \mathrm{C}$ before addition of 2-tridecanone (0.372 g, $1.88 \mathrm{mmol}, 2$ eq.). The solution was then allowed to warm to r.t. and left to stir overnight. The solution was quenched with EtOAc, washed through a short plug of silica with EtOAc and then concentrated in vacuo. Purification by column chromatography (silica, petrol) afforded the title compound $\mathbf{2 b}(0.169 \mathrm{~g}, 69 \%)$ as an inseparable mixture of $Z$ - and $E$ isomers, as a colourless oil. An $E: Z$ ratio of $2.4: 1$ was observed in the ${ }^{1} \mathrm{H}$ NMR spectrum. $\mathrm{R}_{\mathrm{f}} 0.65$ (petrol); $v_{\max }$ (neat) 2956 (s), 2929 (s), 2855 (s), 1667 (C=C,w), $1465(\mathrm{~m}), 1378(\mathrm{~m}) \mathrm{cm}^{-1} ; \delta_{\mathrm{H}}\left(400 \mathrm{MHz} ; \mathrm{CDCl}_{3}\right)$ (both isomers) $5.10(1 \mathrm{H}, \mathrm{t}, J=7.0$, $\mathrm{C}=\mathrm{CH}), 2.02-1.92\left(4 \mathrm{H}, \mathrm{m}, \mathrm{CH}_{2} \mathrm{CCHCH}_{2}\right), 1.66$ and $1.49\left(3 \mathrm{H}, 2 \times \mathrm{s}, \mathrm{Z}-\mathrm{CCH}_{3}\right.$ and $\mathrm{E}-$ $\left.\mathrm{CCH}_{3}\right), 1.40-1.20\left(28 \mathrm{H}, \mathrm{m}, 14 \times \mathrm{CH}_{2}\right), 0.88\left(6 \mathrm{H}\right.$, app. q, $\left.J=7.0,2 \times \mathrm{CH}_{2} \mathrm{CH}_{3}\right) ; \delta_{\mathrm{C}}$ $\left(100 \mathrm{MHz} ; \mathrm{CDCl}_{3}\right)$ (both isomers) 135.4 and $135.1(Z-C=\mathrm{CH}$ and $E-C=\mathrm{CH}), 125.3$ and 124.6 $(Z-\mathrm{C}=C \mathrm{H}$ and $E-\mathrm{C}=C \mathrm{H}), 39.6,31.9,31.8,30.1,29.9,3 \times 29.6,29.5,29.4,2$ $\times 29.3,28.1,28.0,27.9$ and $27.8\left(14 \times \mathrm{CH}_{2}\right), 23.4$ and $15.9\left(\mathrm{Z}-\mathrm{CCH}_{3}\right.$ and $\left.E-\mathrm{CCH}_{3}\right)$, $22.7\left(2 \times \mathrm{CH}_{2} \mathrm{CH}_{3}\right), 14.1\left(2 \times \mathrm{CH}_{2} \mathrm{CH}_{3}\right) ; m / z(\mathrm{EI}) 294\left(\mathrm{M}^{+}, 33 \%\right), 97\left(\mathrm{C}_{7} \mathrm{H}_{13}{ }^{+}, 33\right), 83$ $\left(\mathrm{C}_{6} \mathrm{H}_{11}{ }^{+}, 49\right), 69\left(\mathrm{C}_{5} \mathrm{H}_{9}{ }^{+}, 67\right), 55\left(\mathrm{C}_{4} \mathrm{H}_{7}{ }^{+}, 100\right), 41\left(\mathrm{C}_{3} \mathrm{H}_{5}{ }^{+}, 93\right)$; (Found: $\mathrm{M}^{+}, 294.3292$. $\mathrm{C}_{21} \mathrm{H}_{42}$ requires: $\left.\mathrm{M}^{+}, 294.3287\right)$.

\section{Representative example of a consecutive radical addition/cyclisation/HWE} reaction: 3-(2,2-diphenylvinyl)-4-methyltetrahydrofuran (10)

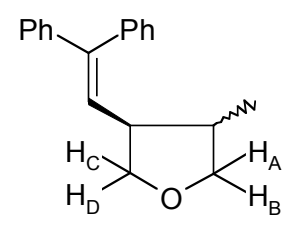

Allyl ether (0.250 g, $2.55 \mathrm{mmol}, 1$ eq. $)$, diethyl thiophosphite $(0.471 \mathrm{~g}, 3.06 \mathrm{mmol}$, 1.2 eq.) and $\operatorname{AIBN}(0.105 \mathrm{~g}, 0.64 \mathrm{mmol}, 0.25$ eq. $)$ in dry THF (20 mL) under $\mathrm{N}_{2}$, were heated to reflux. After $6 \mathrm{~h}$, further AIBN (0.105 g, $0.64 \mathrm{mmol}, 0.25$ eq.) was added. The solution was left at reflux overnight, then cooled to $-78{ }^{\circ} \mathrm{C} .{ }^{\mathrm{s}} \mathrm{BuLi}(6.21 \mathrm{~mL}, 1.23$ $\mathrm{M}$ in cyclohexane, $7.64 \mathrm{mmol}, 3$ eq.) was added, then the solution was allowed to 
warm to $0{ }^{\circ} \mathrm{C}$, re-cooled to $-78{ }^{\circ} \mathrm{C}$ before addition of benzophenone $(0.928 \mathrm{~g}, 5.09$ mmol, 2 eq.). The solution was then allowed to warm to r.t. and left to stir overnight. The solution was quenched with EtOAc, washed through a short plug of silica with EtOAc and then concentrated in vacuo. Purification by column chromatography (silica, petrol/EtOAc, 19:1) afforded the title compound $\mathbf{1 0}(0.620 \mathrm{~g}, 92 \%)$ as an inseparable mixture of cis- and trans-isomers, as a colourless oil. A 3:1 ratio of cis:trans-isomers was observed in the ${ }^{1} \mathrm{H}$ NMR spectrum. $\mathrm{R}_{\mathrm{f}} 0.35$ (petrol/EtOAc, 4:1); $v_{\max }$ (neat) $3079(\mathrm{~m}), 3056(\mathrm{~m}), 3024(\mathrm{~m}), 2962$ (s), 2928 (s), 2857 (s), 1950 (w), 1886 (w), 1809 (w), 1661 (C=C, w), 1598 (m), 1575 (w), 1494 (m), 1445 (s), 1378 (m), $1336(\mathrm{w}), 1240(\mathrm{~m}), 1032(\mathrm{~s}) \mathrm{cm}^{-1} ; \delta_{\mathrm{H}}\left(400 \mathrm{MHz} ; \mathrm{CDCl}_{3}\right.$ ) (cis-isomer) 7.40-7.14 $(10 \mathrm{H}, \mathrm{m}, 10 \times \mathrm{ArCH}), 6.04(1 \mathrm{H}, \mathrm{d}, J=10.5$, cis- $\mathrm{C}=\mathrm{CH}), 3.95-3.87(2 \mathrm{H}, \mathrm{m}$, cis$\left.\mathrm{CH}_{\mathrm{A}} \mathrm{H}_{\mathrm{B}} \mathrm{OC} H_{\mathrm{C}} \mathrm{H}_{\mathrm{D}}\right), 3.69\left(1 \mathrm{H}, \mathrm{dd}, J=8.0\right.$ and 7.0 , cis $\left.-\mathrm{CH}_{\mathrm{C}} H_{\mathrm{D}}\right), 3.53(1 \mathrm{H}, \mathrm{dd}, J=8.0$ and 6.0, cis- $\left.\mathrm{CH}_{\mathrm{A}} H_{\mathrm{B}}\right), 2.97\left(1 \mathrm{H}\right.$, app. dq, $J=10.5$ and 7.0, cis- $\left.\mathrm{CHCH}_{\mathrm{C}} \mathrm{H}_{\mathrm{D}}\right), 2.32(1 \mathrm{H}$, app. septet, $J=7.0$, cis- $\left.\mathrm{CHCH}_{3}\right), 1.08\left(3 \mathrm{H}, \mathrm{d}, J=7.0\right.$, cis- $\left.\mathrm{CH}_{3}\right) ; \delta_{\mathrm{C}}\left(100 \mathrm{MHz} ; \mathrm{CDCl}_{3}\right)$ (cis-isomer) $143.7,142.3$ and 140.0 (2 × cis-ArC, cis- $C=\mathrm{CH}), 129.8,128.8,128.3$, 127.2, 127.1, 127.0 and $126.8(10 \times \mathrm{ArCH}, \mathrm{C}=\mathrm{CH}), 75.1$ and 72.9 (cis$\left.\mathrm{CH}_{\mathrm{A}} \mathrm{H}_{\mathrm{B}} \mathrm{OCH}_{\mathrm{C}} \mathrm{H}_{\mathrm{D}}\right), 43.0($ cis- $\mathrm{C}=\mathrm{CHCH}), 38.2\left(\right.$ cis $\left.-\mathrm{CH}_{3} \mathrm{CH}\right), 13.9\left(\right.$ cis- $\left.\mathrm{CH}_{3}\right) ; m / z(\mathrm{CI}$, $\left.\mathrm{NH}_{3}\right) 282\left(\mathrm{M}+\mathrm{NH}_{4}^{+}, 39 \%\right), 265\left(\mathrm{M}+\mathrm{H}^{+}, 100\right), 207\left(\mathrm{Ph}_{2} \mathrm{CCHCHCH}_{2}{ }^{+}, 46\right), 180$ $\left(\mathrm{Ph}_{2} \mathrm{CCH}_{2}{ }^{+}, 56\right)$; (Found: $\mathrm{M}+\mathrm{H}^{+}, 265.1590 . \mathrm{C}_{19} \mathrm{H}_{20} \mathrm{O}$ requires: $\left.\mathrm{M}+\mathrm{H}^{+}, 265.1592\right)$.

The presence of the trans-isomer was indicated by: $\delta_{\mathrm{H}}\left(400 \mathrm{MHz} ; \mathrm{CDCl}_{3}\right) 5.92(1 \mathrm{H}$, $\mathrm{d}, J=10.0$, trans $-\mathrm{C}=\mathrm{C} H), 4.03\left(1 \mathrm{H}\right.$, app. t, $J=8.0$, trans $\left.-\mathrm{CH}_{\mathrm{A}} \mathrm{H}_{\mathrm{B}}\right), 3.95-3.87(1 \mathrm{H}, \mathrm{m}$, trans $\left.-\mathrm{CH}_{\mathrm{C}} \mathrm{H}_{\mathrm{D}}\right), 3.60\left(1 \mathrm{H}\right.$, app. t, $J=8.5$, trans $\left.-\mathrm{CH}_{\mathrm{C}} H_{\mathrm{D}}\right), 3.28(1 \mathrm{H}$, app. t, $J=8.5$, trans $\left.-\mathrm{CH}_{\mathrm{A}} H_{\mathrm{B}}\right), 2.52\left(1 \mathrm{H}\right.$, app. q, $J=9.0$, trans $\left.-\mathrm{CHCH}_{\mathrm{C}} \mathrm{H}_{\mathrm{D}}\right), 2.20-2.10(1 \mathrm{H}, \mathrm{m}$, trans$\left.\mathrm{CHCH}_{3}\right), 0.94\left(3 \mathrm{H}, \mathrm{d}, J=7.0\right.$, trans $\left.-\mathrm{CH}_{3}\right) ; \delta_{\mathrm{C}}\left(100 \mathrm{MHz} ; \mathrm{CDCl}_{3}\right) 144.2,141.9$ and $139.9(2 \times$ trans $-\mathrm{ArC}$, trans $-\mathrm{C}=\mathrm{CH}), 75.2$ and $73.4\left(\right.$ trans $\left.-\mathrm{CH}_{\mathrm{A}} \mathrm{H}_{\mathrm{B}} \mathrm{OCH}_{\mathrm{C}} \mathrm{H}_{\mathrm{D}}\right), 47.9$ (trans $-\mathrm{C}=\mathrm{CHCH}), 41.7$ (trans $\left.-\mathrm{CH}_{3} \mathrm{CH}\right), 15.1$ (trans $\left.-\mathrm{CH}_{3}\right)$.

Representative example of a consecutive radical addition/alkylation/HWE reaction: 1-(1-methyloctylidene)cyclohexane (13b)<smiles>CCCCCCCC(C)=C1CCCCC1</smiles> 
1-Octene (0.250 g, $1.98 \mathrm{mmol}, 1$ eq.), diethyl thiophosphite (0.366 g, $2.38 \mathrm{mmol}, 1.2$ eq.) and $\mathrm{AIBN}(0.081 \mathrm{~g}, 0.50 \mathrm{mmol}, 0.25$ eq. $)$ in dry THF $(20 \mathrm{~mL})$ under $\mathrm{N}_{2}$, were heated to reflux. After $6 \mathrm{~h}$, further AIBN (0.081 g, $0.50 \mathrm{mmol}, 0.25$ eq.) was added. The solution was left at reflux overnight, then cooled to $-78{ }^{\circ} \mathrm{C} .{ }^{\mathrm{s}} \mathrm{BuLi}(4.83 \mathrm{~mL}, 1.23$ $\mathrm{M}$ in cyclohexane, $5.94 \mathrm{mmol}, 3$ eq.) was added, then the solution was allowed to warm to $0{ }^{\circ} \mathrm{C}$, re-cooled to $-78{ }^{\circ} \mathrm{C}$ before addition of iodomethane $(0.281 \mathrm{~g}, 1.98$ mmol, 1 eq.). The solution was then allowed to warm to r.t. and left to stir overnight. The solution was cooled to $-78{ }^{\circ} \mathrm{C}$ before adding ${ }^{\mathrm{s}} \mathrm{BuLi}(3.22 \mathrm{~mL}, 1.23 \mathrm{M}$ in cyclohexane, $3.96 \mathrm{mmol}, 2$ eq.), the solution was allowed to warm to $0{ }^{\circ} \mathrm{C}$, re-cooled to $-78{ }^{\circ} \mathrm{C}$ before addition of cyclohexanone $(0.389 \mathrm{~g}, 3.96 \mathrm{mmol}, 2 \mathrm{eq}$. $)$. The solution was then allowed to warm to r.t. and left to stir overnight. The solution was quenched with EtOAc, washed through a short plug of silica with EtOAc and then concentrated in vacuo. Purification by column chromatography (silica, petrol) afforded the title compound 13b as an inseparable mixture with 1-octylidenecyclohexane $\mathbf{2 c}$ (in the ratio of 12.6:1, respectively, from the ${ }^{1} \mathrm{H}$ NMR spectrum) as a yellow oil $(0.267 \mathrm{~g}): \mathrm{R}_{\mathrm{f}}$ 0.65 (petrol); $v_{\max }$ (neat) $2920(\mathrm{~s}), 2854(\mathrm{~s}), 1658(\mathrm{C}=\mathrm{C}, \mathrm{w}), 1446(\mathrm{~m}), 1378$ (s), 1260 $(\mathrm{m}), 1236(\mathrm{~m}) \mathrm{cm}^{-1}$. The presence of $\mathbf{1 3 b}$ was indicated by: $\delta_{\mathrm{H}}\left(400 \mathrm{MHz} ; \mathrm{CDCl}_{3}\right)$ $2.13\left(4 \mathrm{H}, \mathrm{t}, J=6.0,2 \times \mathrm{CH}_{2} \mathrm{C}=\mathrm{CCH}_{3}\right), 2.01\left(2 \mathrm{H}, \mathrm{t}, J=7.0, \mathrm{C}=\mathrm{C}\left(\mathrm{CH}_{3}\right) \mathrm{CH}_{2}\right), 1.64(3 \mathrm{H}$, s, $\left.\mathrm{C}=\mathrm{CCH}_{3}\right), 1.57-1.44\left(6 \mathrm{H}, \mathrm{m}, \mathrm{CH}_{2} \mathrm{CH}_{2} \mathrm{CH}_{2} \mathrm{CH}_{2} \mathrm{C}=\mathrm{CCH}_{3}\right), 1.36-1.21(10 \mathrm{H}, \mathrm{m}, 5 \times$ $\left.\mathrm{CH}_{2}\right), 0.89\left(3 \mathrm{H}, \mathrm{t}, J=7.0, \mathrm{CH}_{2} \mathrm{CH}_{2} \mathrm{CH}_{3}\right) ; \delta_{\mathrm{C}}\left(100 \mathrm{MHz} ; \mathrm{CDCl}_{3}\right) 132.5\left(C=\mathrm{CCH}_{3}\right)$, $124.8\left(\mathrm{C}=\mathrm{CCH}_{3}\right), 34.1,31.9,30.6,30.3,29.6,29.3,28.8,28.4,28.1$ and $27.0(10 \times$ $\left.\mathrm{CH}_{2}\right), 22.7\left(\mathrm{CH}_{2} \mathrm{CH}_{2} \mathrm{CH}_{3}\right), 17.9\left(\mathrm{C}=\mathrm{CCH}_{3}\right), 14.1\left(\mathrm{CH}_{2} \mathrm{CH}_{2} \mathrm{CH}_{3}\right) ; m / z(\mathrm{EI}) 208\left(\mathrm{M}^{+}\right.$, 35\%), $123\left(\mathrm{C}_{9} \mathrm{H}_{15}{ }^{+}, 51\right), 109\left(\mathrm{C}_{8} \mathrm{H}_{13}{ }^{+}, 97\right), 81\left(\mathrm{C}_{6} \mathrm{H}_{9}{ }^{+}, 100\right), 67\left(\mathrm{C}_{5} \mathrm{H}_{7}{ }^{+}, 74\right), 55\left(\mathrm{C}_{4} \mathrm{H}_{7}{ }^{+}\right.$, 44), $41\left(\mathrm{C}_{3} \mathrm{H}_{5}^{+}, 45\right)$; (Found: $\mathrm{M}^{+}, 208.2197 . \mathrm{C}_{15} \mathrm{H}_{28}$ requires: $\left.\mathrm{M}^{+}, 208.2191\right)$.

\section{Representative example of a consecutive radical addition/acylation reaction:}

ethyl 2-(diethoxyphosphorothioyl)nonanoate (15a)

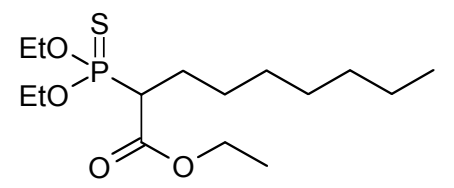

1-Octene (0.250 g, $2.23 \mathrm{mmol}, 1$ eq.), diethyl thiophosphite (0.412 g, $2.67 \mathrm{mmol}, 1.2$ eq.) and AIBN (0.091 g, $0.56 \mathrm{mmol}, 0.25$ eq.) in dry THF $(20 \mathrm{~mL})$ were heated to reflux. After $6 \mathrm{~h}$, further AIBN (0.091 g, $0.56 \mathrm{mmol}, 0.25$ eq. $)$ was added and the 
solution was left to stir overnight. The solution was then cooled to $-78{ }^{\circ} \mathrm{C}$, before addition of LDA (3.71 mL, 1.8 M in heptane/THF/ethylbenzene, $6.68 \mathrm{mmol}, 3$ eq.). After $1 \mathrm{~h}$, diethyl carbonate $(0.40 \mathrm{~mL}, 3.34 \mathrm{mmol}, 1.5$ eq. $)$ was added then the solution was allowed to warm to r.t. and left to stir overnight. The reaction was quenched with water and extracted with EtOAc. The organic portions were combined, dried $\left(\mathrm{Na}_{2} \mathrm{SO}_{4}\right)$, filtered and concentrated in vacuo. Purification by column chromatography (silica, petrol/EtOAc, 19:1) afforded the title compound 15a (0.649 g, 86\%) as a yellow oil. Purification by column chromatography (silica, petrol/EtOAc, 19:1) afforded the title compound $15 \mathrm{a}(1.852 \mathrm{~g}, 97 \%)$ as a colourless oil. $\mathrm{R}_{\mathrm{f}} 0.40$ (petrol/EtOAc, 9:1); $v_{\max }$ (neat) 2980 (s), 2957 (s), 2927 (s), 2857 (m), 1736 (C=O, s), 1465 (m), 1389 (m), 1367 (m), 1329 (m), 1254 (m), 1156 (s), 1115 (s), 1096 (s), 1045 (s), $1025(\mathrm{P}-\mathrm{OEt}, \mathrm{s}) \mathrm{cm}^{-1} ; \delta_{\mathrm{H}}\left(400 \mathrm{MHz} ; \mathrm{CDCl}_{3}\right) 4.27-4.07\left(6 \mathrm{H}, \mathrm{m}, 3 \times \mathrm{OCH}_{2} \mathrm{CH}_{3}\right)$, $3.04(1 \mathrm{H}, \mathrm{ddd}, J=19.0,11.0$ and 3.5, $\mathrm{PCH}), 2.02-1.80\left(2 \mathrm{H}, \mathrm{m}, \mathrm{PCHCH}_{2}\right), 1.39-1.20$ $\left(10 \mathrm{H}, \mathrm{m}, 5 \times \mathrm{CH}_{2}\right), 1.31\left(3 \mathrm{H}, \mathrm{t}, J=7.0, \mathrm{OCH}_{2} \mathrm{CH}_{3}\right), 1.30\left(3 \mathrm{H}, \mathrm{t}, J=7.0, \mathrm{OCH}_{2} \mathrm{CH}_{3}\right)$, $1.29\left(3 \mathrm{H}, \mathrm{t}, J=7.0, \mathrm{OCH}_{2} \mathrm{CH}_{3}\right), 0.89\left(3 \mathrm{H}, \mathrm{t}, J=7.0, \mathrm{CH}_{2} \mathrm{CH}_{3}\right) ; \delta_{\mathrm{C}}\left(100 \mathrm{MHz} ; \mathrm{CDCl}_{3}\right)$ $169.0\left(\mathrm{~d}, J=5.0, \mathrm{CO}_{2} \mathrm{CH}_{2} \mathrm{CH}_{3}\right), 63.3$ (d, $\left.J=7.0, \mathrm{POCH}_{2} \mathrm{CH}_{3}\right), 63.1$ (d, $J=7.0$, $\left.\mathrm{POCH}_{2} \mathrm{CH}_{3}\right), 61.2\left(\mathrm{CO}_{2} \mathrm{CH}_{2} \mathrm{CH}_{3}\right), 53.0(\mathrm{~d}, J=101.0, \mathrm{PCH}), 31.7,29.1$ and $29.0(3 \times$ $\left.\mathrm{CH}_{2}\right), 28.2\left(\mathrm{~d}, J=16.0, \mathrm{PCHCH}_{2} \mathrm{CH}_{2}\right), 27.3\left(\mathrm{~d}, J=4.0, \mathrm{PCHCH}_{2}\right), 22.6$ $\left(\mathrm{CH}_{2} \mathrm{CH}_{2} \mathrm{CH}_{3}\right), 16.2$ (d, $\left.J=7.0, \mathrm{POCH}_{2} \mathrm{CH}_{3}\right), 16.1$ (d, $\left.J=7.0, \mathrm{POCH}_{2} \mathrm{CH}_{3}\right), 14.2$ $\left(\mathrm{CO}_{2} \mathrm{CH}_{2} \mathrm{CH}_{3}\right), 14.1\left(\mathrm{CH}_{2} \mathrm{CH}_{2} \mathrm{CH}_{3}\right) ; m / z\left(\mathrm{CI}, \mathrm{NH}_{3}\right) 339\left(\mathrm{M}+\mathrm{H}^{+}, 100 \%\right)$; (Found: $\mathrm{M}+\mathrm{H}^{+}, 339.1755 . \mathrm{C}_{15} \mathrm{H}_{31} \mathrm{O}_{4}$ PS requires: $\left.\mathrm{M}+\mathrm{H}^{+}, 339.1759\right)$.

\section{Representative example of a HWE reaction of an $\alpha$-acylated phosphonothioate:} ethyl (E)-2-heptyl-3-methyl-2-butenedioate (16)

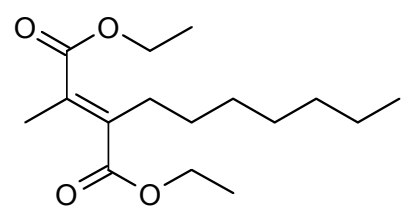

To ethyl 2-(diethoxyphosphorothioyl)nonanoate $15 \mathrm{a}(0.150 \mathrm{~g}, 0.44 \mathrm{mmol}, 1$ eq.) in dry THF $(20 \mathrm{~mL})$ at $-78{ }^{\circ} \mathrm{C}$, under $\mathrm{N}_{2}$, was added ${ }^{\mathrm{s}} \mathrm{BuLi}(0.89 \mathrm{~mL}, 1.0 \mathrm{M}$ in cyclohexane, $0.88 \mathrm{mmol}, 2$ eq.). The solution was allowed to warm to $0{ }^{\circ} \mathrm{C}$, then cooled to $-78{ }^{\circ} \mathrm{C}$ before addition of ethyl pyruvate $(0.10 \mathrm{~mL}, 0.88 \mathrm{mmol}, 2$ eq. $)$. The solution was allowed to warm to r.t. and left to stir overnight. The reaction was quenched with water and extracted with EtOAc. The organic portions were combined, 
dried $\left(\mathrm{Na}_{2} \mathrm{SO}_{4}\right)$, filtered and concentrated in vacuo. Purification by column chromatography (silica, petrol/EtOAc, 19:1) afforded the title compound 16 (0.096 g, 87\%) as a yellow oil. $\mathrm{R}_{\mathrm{f}} 0.30$ (petrol/EtOAc, 9:1); $v_{\max }$ (neat) 2957 (s), 2927 (s), 2857 (s), $1722(\mathrm{C}=\mathrm{O}, \mathrm{s}), 1644(\mathrm{C}=\mathrm{C}, \mathrm{m}), 1464$ (m), 1367 (m), 1301 (s), 1260 (s), 1178 (s), 1098 (s), 1027 (s) cm ${ }^{-1} ; \delta_{\mathrm{H}}\left(400 \mathrm{MHz} ; \mathrm{CDCl}_{3}\right) 4.22\left(2 \mathrm{H}, \mathrm{q}, J=7.0, \mathrm{OCH}_{2} \mathrm{CH}_{3}\right), 4.20$ $\left(2 \mathrm{H}, \mathrm{q}, J=7.0, \mathrm{OCH}_{2} \mathrm{CH}_{3}\right), 2.33\left(2 \mathrm{H}, \mathrm{t}, J=7.5, \mathrm{C}=\mathrm{CCH}_{2}\right), 1.94\left(3 \mathrm{H}, \mathrm{s}, \mathrm{CH}_{3} \mathrm{C}=\mathrm{C}\right)$, 1.48-1.40 (2H, m, C= $\left.\mathrm{CCH}_{2} \mathrm{CH}_{2}\right), 1.34-1.22\left(8 \mathrm{H}, \mathrm{m}, 4 \times \mathrm{CH}_{2}\right), 1.30(3 \mathrm{H}, \mathrm{t}, J=7.0$, $\left.\mathrm{OCH}_{2} \mathrm{CH}_{3}\right), 1.29\left(3 \mathrm{H}, \mathrm{t}, J=7.0, \mathrm{OCH}_{2} \mathrm{CH}_{3}\right), 0.88\left(3 \mathrm{H}, \mathrm{t}, J=7.0, \mathrm{CH}_{2} \mathrm{CH}_{2} \mathrm{CH}_{3}\right) ; \delta_{\mathrm{C}}$ $\left(100 \mathrm{MHz} ; \mathrm{CDCl}_{3}\right) 169.6$ and $168.5\left(2 \times \mathrm{CO}_{2} \mathrm{CH}_{2} \mathrm{CH}_{3}\right), 139.6$ and 131.4 $\left(\mathrm{CH}_{3} \mathrm{C}=\mathrm{CCH}_{2}\right), 61.0$ and $60.9\left(2 \times \mathrm{CO}_{2} \mathrm{CH}_{2} \mathrm{CH}_{3}\right), 31.7,30.2,29.4,29.0$ and $27.7(5 \times$ $\left.\mathrm{CH}_{2}\right), 22.6\left(\mathrm{CH}_{2} \mathrm{CH}_{2} \mathrm{CH}_{3}\right), 15.0\left(\mathrm{CH}_{3} \mathrm{C}=\mathrm{C}\right), 14.1$ and $2 \times 14.0\left(2 \times \mathrm{CO}_{2} \mathrm{CH}_{2} \mathrm{CH}_{3}\right.$, $\left.\mathrm{CH}_{2} \mathrm{CH}_{2} \mathrm{CH}_{3}\right) ; m / z\left(\mathrm{CI}, \mathrm{NH}_{3}\right) 302\left(\mathrm{M}+\mathrm{NH}_{4}{ }^{+}, 12 \%\right), 285\left(\mathrm{M}+\mathrm{H}^{+}, 31\right), 239\left(\mathrm{C}_{14} \mathrm{H}_{23} \mathrm{O}_{3}{ }^{+}\right.$, 100); (Found: $\mathrm{M}+\mathrm{H}^{+}, 285.2064 . \mathrm{C}_{16} \mathrm{H}_{28} \mathrm{O}_{4}$ requires: $\mathrm{M}+\mathrm{H}^{+}, 285.2066$ ).

Example of a consecutive radical addition/acylation/HWE reaction: ethyl (E)-2heptyl-3-phenyl-2-propenoate (17)

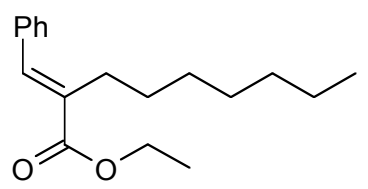

1-Octene (0.250 g, $2.23 \mathrm{mmol}, 1$ eq.), diethyl thiophosphite (0.412 g, $2.67 \mathrm{mmol}, 1.2$ eq.) and AIBN (0.091 g, $0.56 \mathrm{mmol}, 0.25$ eq.) in dry THF $(20 \mathrm{~mL})$ were heated to reflux. After $6 \mathrm{~h}$, further AIBN $(0.091 \mathrm{~g}, 0.56 \mathrm{mmol}, 0.25$ eq. $)$ was added and the mixture was left to stir overnight. The mixture was then cooled to $-78{ }^{\circ} \mathrm{C}$ before addition of LDA (3.10 mL, 1.8 $\mathrm{M}$ in heptane/THF/ethylbenzene, $5.57 \mathrm{mmol}, 2.5$ eq.). The solution was left to stir for $1 \mathrm{~h}$, then diethyl carbonate $(0.28 \mathrm{~mL}, 2.34 \mathrm{mmol}, 1.05$ eq.) was added and the solution was allowed to warm to r.t. then left to stir overnight. The solution was then cooled to $-78{ }^{\circ} \mathrm{C}$ before addition of LDA $(2.48 \mathrm{~mL}, 1.8 \mathrm{M}$ in heptane/THF/ethylbenzene, $4.46 \mathrm{mmol}, 2.0$ eq.). The solution was left to stir for $1 \mathrm{~h}$, then benzaldehyde ( $0.45 \mathrm{~mL}, 4.46 \mathrm{mmol}, 2$ eq.) was added and the solution was then allowed to warm to r.t. then left to stir overnight. The reaction was quenched with water and extracted with EtOAc. The organic portions were combined, dried $\left(\mathrm{Na}_{2} \mathrm{SO}_{4}\right)$, filtered and concentrated in vacuo. Purification by column chromatography (silica, petrol/EtOAc, 37:3) afforded the title compound $17(0.110 \mathrm{~g}, 90 \%)$ as a 
colourless oil. $\mathrm{R}_{\mathrm{f}} 0.60$ (petrol/EtOAc, 9:1); $v_{\max }$ (neat) 3082 (w), 3058 (w), 3025 (w), 2956 (s), 2927 (s), 2858 (m), 1709 (C=O, s), 1631 (C=C, w), 1494 (w), 1463 (m), $1368(\mathrm{~m}), 1235$ (s), 1201 (s), 1175 (m), $1130(\mathrm{~s}) \mathrm{cm}^{-1} ; \delta_{\mathrm{H}}\left(400 \mathrm{MHz} ; \mathrm{CDCl}_{3}\right) 7.64$ $\left(1 \mathrm{H}, \mathrm{s}, \mathrm{CH}=\mathrm{CCH}_{2}\right), 7.42-7.28(5 \mathrm{H}, \mathrm{m}, 5 \times \mathrm{ArCH}), 4.27\left(2 \mathrm{H}, \mathrm{q}, J=7.0, \mathrm{OCH}_{2} \mathrm{CH}_{3}\right)$, $2.50\left(2 \mathrm{H}, \mathrm{t}, J=8.0, \mathrm{CH}=\mathrm{CCH}_{2}\right), 1.58-1.50\left(2 \mathrm{H}, \mathrm{m}, \mathrm{CH}=\mathrm{CCH}_{2} \mathrm{CH}_{2}\right), 1.38-1.22(8 \mathrm{H}$, $\left.\mathrm{m}, 4 \times \mathrm{CH}_{2}\right), 1.35\left(3 \mathrm{H}, \mathrm{t}, J=7.0, \mathrm{OCH}_{2} \mathrm{CH}_{3}\right), 0.88\left(3 \mathrm{H}, \mathrm{t}, J=7.0, \mathrm{CH}_{2} \mathrm{CH}_{2} \mathrm{CH}_{3}\right) ; \delta_{\mathrm{C}}$ $\left(100 \mathrm{MHz} ; \mathrm{CDCl}_{3}\right) 168.5\left(\mathrm{CO}_{2} \mathrm{CH}_{2} \mathrm{CH}_{3}\right), 138.4\left(\mathrm{CH}=\mathrm{CCH}_{2}\right), 135.9\left(\mathrm{ArCCH}=\mathrm{CCH}_{2}\right)$, $134.0\left(\mathrm{CH}=\mathrm{CCH}_{2}\right), \quad 129.2 \quad\left(2 \times \quad \mathrm{ArC} \mathrm{HArCCH}=\mathrm{CCH}_{2}\right), \quad 128.4 \quad(2 \times$ $\left.\operatorname{ArCHArCHArCCH}=\mathrm{CCH}_{2}\right), \quad 128.2 \quad\left(\mathrm{ArCHArCHArCHArCCH}=\mathrm{CCH}_{2}\right), \quad 60.7$ $\left(\mathrm{CO}_{2} \mathrm{CH}_{2} \mathrm{CH}_{3}\right), 31.7,29.7,29.2,29.0$ and $27.5\left(5 \times \mathrm{CH}_{2}\right), 22.6\left(\mathrm{CH}_{2} \mathrm{CH}_{2} \mathrm{CH}_{3}\right), 14.3$ $\left(\mathrm{CO}_{2} \mathrm{CH}_{2} \mathrm{CH}_{3}\right), 14.1\left(\mathrm{CH}_{2} \mathrm{CH}_{2} \mathrm{CH}_{3}\right) ; \mathrm{m} / z\left(\mathrm{CI}, \mathrm{NH}_{3}\right) 292\left(\mathrm{M}+\mathrm{NH}_{4}{ }^{+}, 30 \%\right), 275\left(\mathrm{M}+\mathrm{H}^{+}\right.$, 100\%); (Found: $\mathrm{M}+\mathrm{H}^{+}, 275.2013 . \mathrm{C}_{18} \mathrm{H}_{26} \mathrm{O}_{2}$ requires: $\mathrm{M}+\mathrm{H}^{+}, 275.2011$ ). 

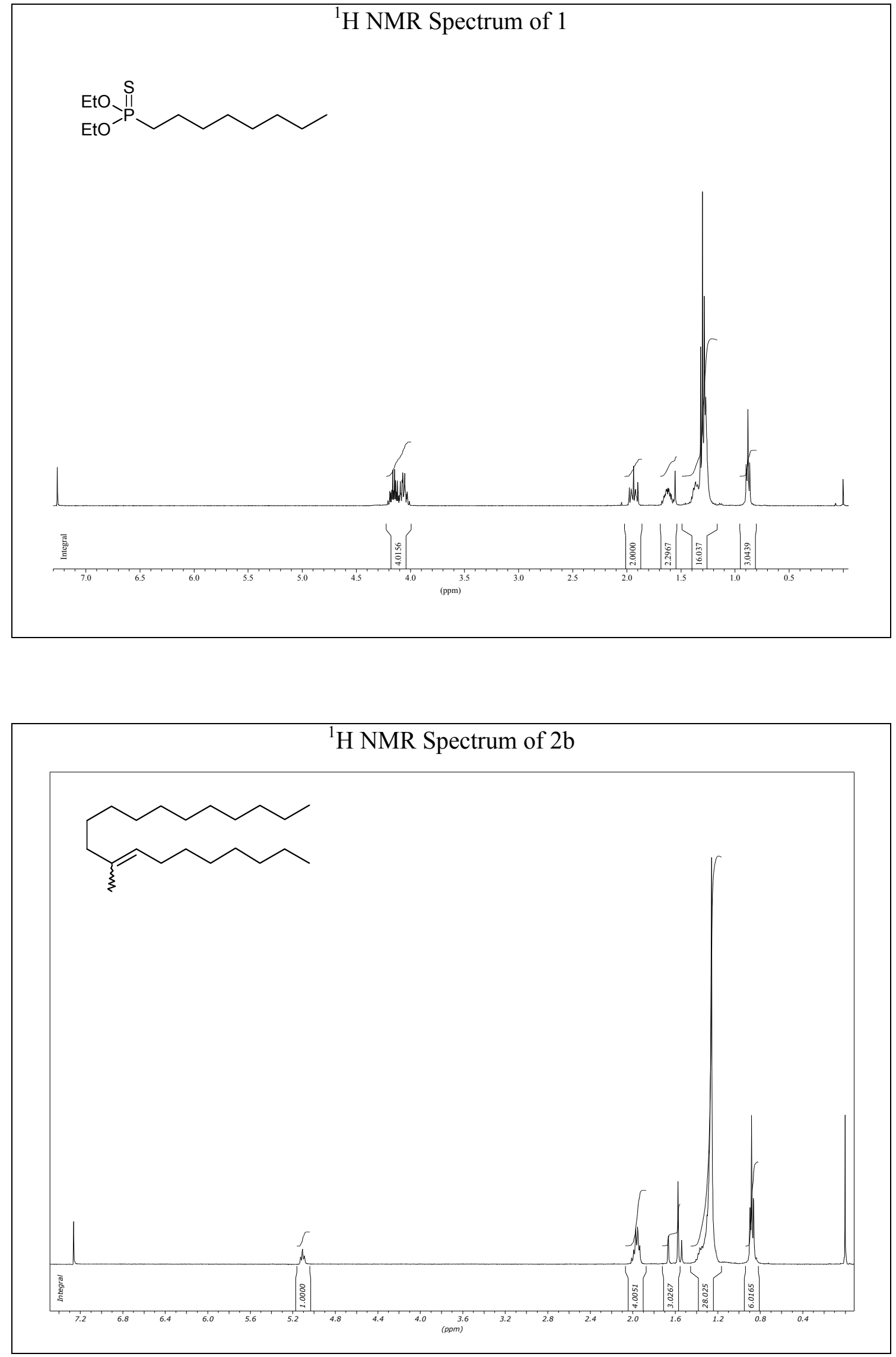

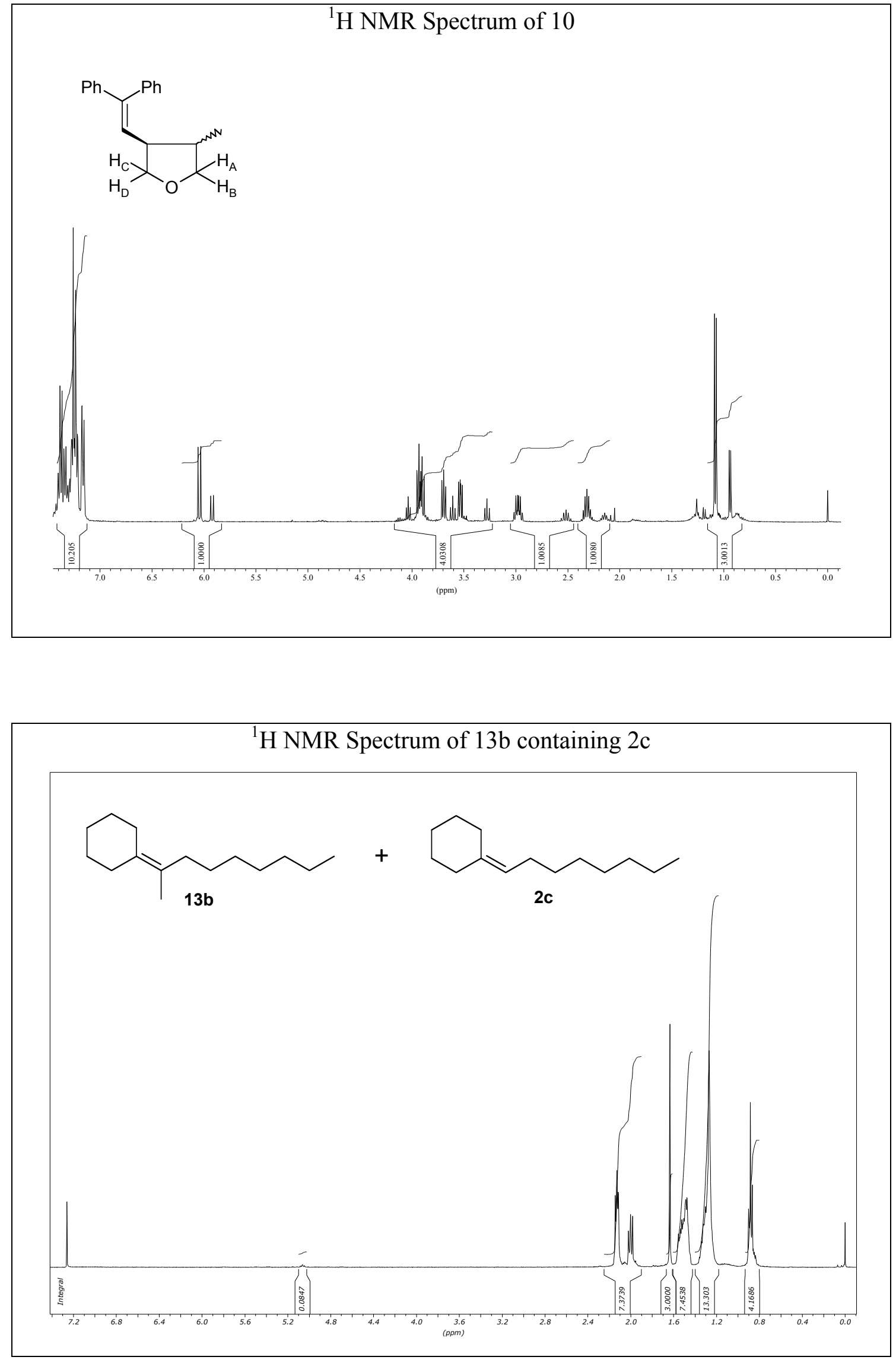

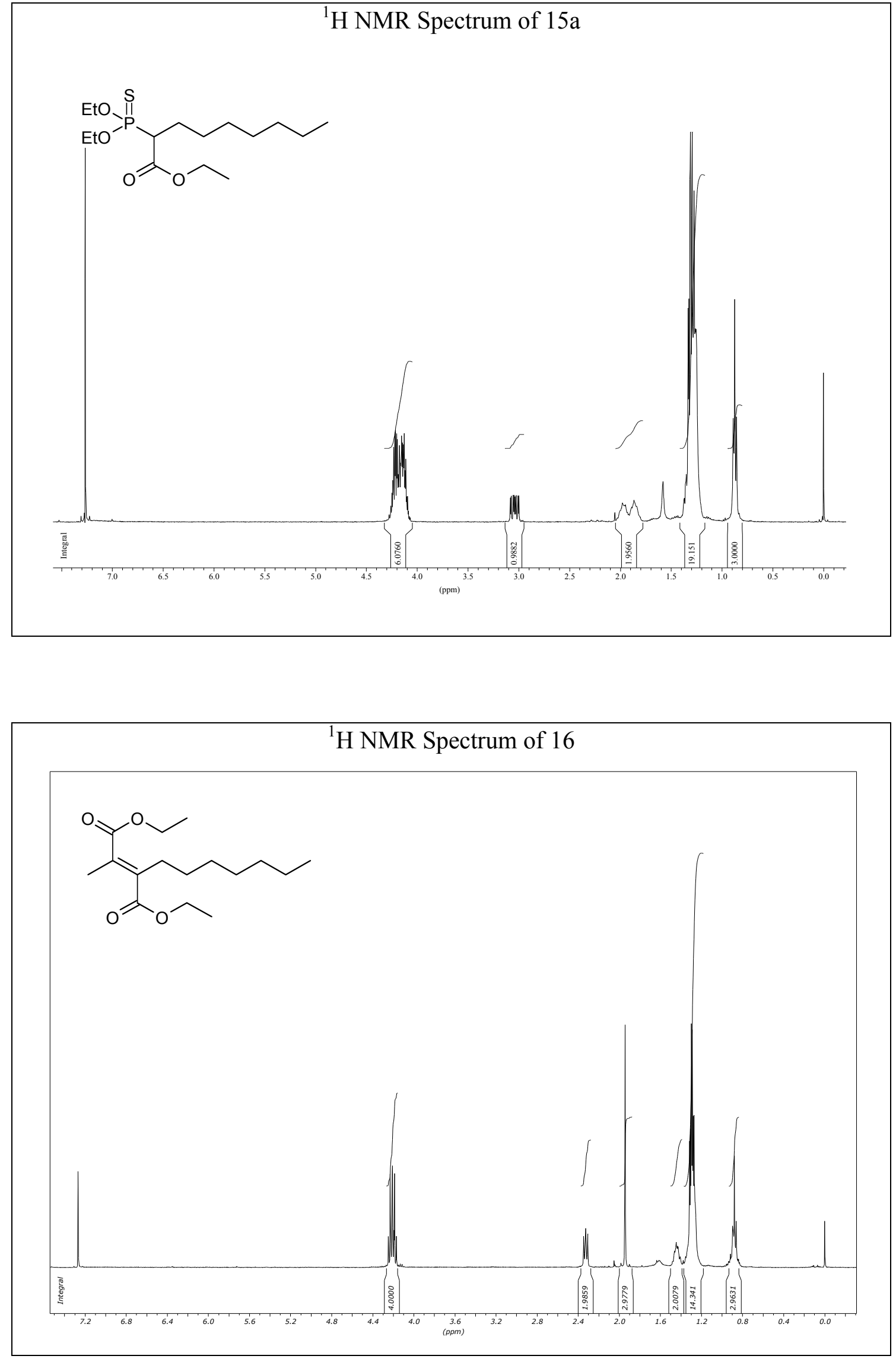


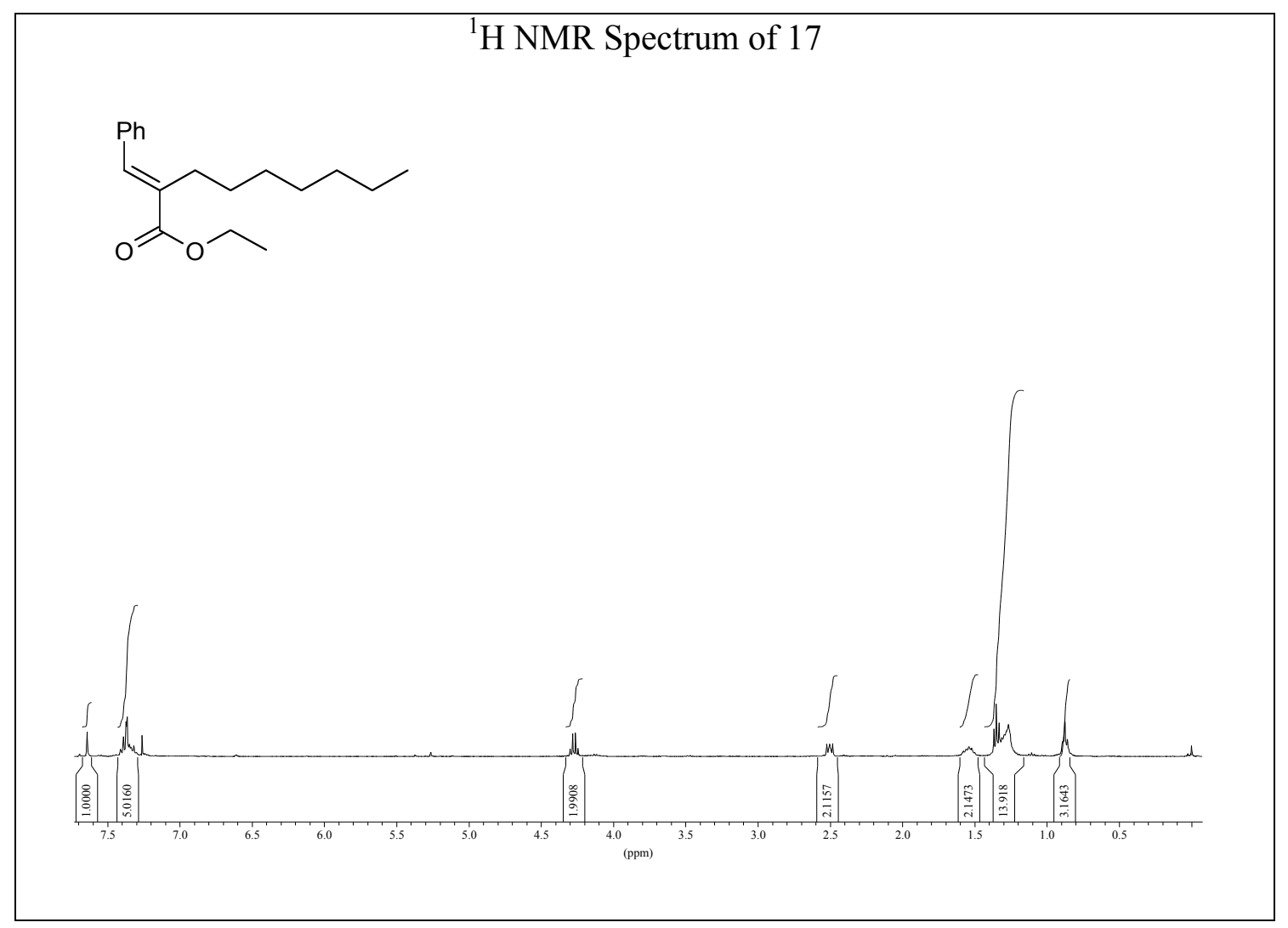

11 